\title{
Chiropractic Discourse: Adjusting Spines, Locating Subjects
}

\author{
Charles Horn \\ Ministry of Aboriginal Affairs \\ Province of British Columbia
}

This paper examines the role of written communication in medical settings. The analysis focusses on a pamphlet written for chiropractors, for use with new patients. It is argued that the document's central role is to construct a textual identity for chiropractors, which identifies with and differs from that of conventional physicians. The analysis reveals the discursive construction of the chiropractor in chiropractic discourse of the spine, which functions as both source and cure for pathology, and as the special object of chiropractic knowledge. Conventional physicians are in turn constructed through their failure to grasp the role of the spine. A third textual identity is also developed, that of the chiropractice patient. This article discusses both the specific textual resources used in these constructions, and the general role of discourse in medical settings.

THIS PAPER DISCUSSES a specific example of the written information chiropractors give to their patients. In what follows, I analyze that information as a vehicle for a whole host of social messages, the general thrust of which is to construct the social location of doctors of chiropractic, in particular, their relationship to other medical doctors and to potential patients. I examine one particular document, a pamphlet entitled "To the New Patient" (attached as Appendix A). ${ }^{1}$ I suggest that the pamphlet's principal effect is to construct

1 The document is produced by the "Parker Chiropractic Research Foundation," and is distributed to chiropractors in Canada and the United States. A relatively limited examination of the documents in a number of chiropractors' offices indicates that the selected document is not untypical of the public health education information made available by chiropractors, although the actual disposition of such documents by chiropractors in medical encounters warrants further research. (Continued next page.) 
a chiropractic identity, an identity which both draws from and repudiates established definitions of physicians' roles. The document constructs other identities as well, including that of the reader and that of conventional physicians, and it is the effort to construct these identities that gives the document, to borrow a phrase, its texture (Halliday and Hasan, 1976). So the text under investigation here establishes a range of identities, or "subject-positions," or what Kaja Silverman, following Benveniste, has called the "spoken subject" (Silverman, 1983): the chiropractor as a legitimate medical professional; members of the conventional medical community as ineffective and parasitic practitioners, yet in possession of a powerful discourse; and the reader as unwell, and by virtue of this, a (potential) chiropractic patient. The analysis focusses on these three constructions, specifying their role in the document and arguing for the centrality of such discursive maneuvers to the practice of chiropractic.

And, in the process of that analysis, we discover that the identity of subjects in the document is derived not just from the specific medical status of their spinal cords, which one might expect, but more importantly, from the subjects' specificknowledge of the spinal cord: its role in disease, its essential properties, and its relation to the remainder of the body.

Finally, I briefly explore in the paper the paradoxical nature of the text under discussion: while overtly rejecting the institutions of medicine, the document relies upon the medico-technical discourse that underlies those institutions to persuade readers of the legitimacy of chiropractic care, and to provide a base upon which to build its subject positions. There is thus a tension created in the text between accepting a substantive component of medical discourse and rejecting medical practitioners and their institutions.

The literature which doctors of chiropractic produce can thus be read as a field of strategic negotiation, via the construction of subjects, for a qualified place within the medical community, and for acceptance by the larger realm of medical subjects, construed as potential recipients of chiropractic care.

\section{Chiropractic Documents}

I have chosen for the analysis to draw from a pamphlet produced by a chiropractic research foundation. A pamphlet is not usually the type of text

In the absence of detailed research on such documents, the analysis proceeds on the assumption that this document is roughly representative of key elements of chiropractic discourse, and that explanations of chiropractic have a role in the way chiropractors use discourse to accomplish social relations. 
employed by analysts to understand doctor-patient relations. The growing body of literature on the topic by and large concerns itself with conversations between physicians and their patients (Bax, 1986; Candlin and Lucas, 1986; Cicourel, 1987, 1982; Coulthard and Ashbym 1976; Fisher, 1983; Waitzkin, 1976). There have been some studies of, for instance, the constraints placed upon doctor-patient interactions by bureaucratic and documentary requirements (see Collins, 1987; Freeman, 1987; Silverman, 1987), but little work has been done on documents created and used by medical professionals in their interactions with patients. So one aim of this study is to contribute towards our understanding of the social role of such documents.

A second limitation of the existing literature has been its relative silence on the ways in which the agents involved in the medical encounter are discursively constituted (and not merely reflected) through their participation in the communicative process. This suggests at least some of the motivation for what follows, which is an attempt to explore, in a particular instance, the rhetorical construction of selves and the social relations between them, an approach which is in contrast to the more usual examination of the finished rhetorical product (for example, the stable, continuous, pre-existing individual of classical social theory) (Battaglia, 1995).

For these reasons, the analysis proceeds by means of a close textual reading of a specific document, paying attention to its lexico-grammatical features, in the hope that such a focus will provide a glimpse of the ways selves and relations are constituted in the mundane exchanges of everyday life. With respect to the focus on the document's formal features, I cite in my defence Pierre Bourdieu, who argues throughout much of his work that "[f]orm and the information it imparts condense and symbolize the entire structure of the social relation from which they derive their existence and their efficacy" (1991:80). As a linguistic product designed in anticipation of its circulation in a discursive economy, the pamphlet is marked by the conditions of its production, "even at a grammatical level." Through an attentive reading of the document's form, I hope to reveal something about the social context of its production, and hence of its reception.

The document under the microscope here is entitled, as noted earlier, "To the New Patient." The document is in standard, semi-gloss pamphlet form, and ostensibly offers an introduction to and an explanation of chiropractic, by way of 15 paragraphs of prose, a text box, and three medical diagrams. In its general form, its layout, and its location, "To the New Patient" resembles the kind of pamphlet which sits on open racks in the waiting rooms 
of doctors, dentists, and other health professionals. Such documents, of which this is but one type, are typically displayed in a way that indicates their public nature. Displaying themselves in visible, accessible ways and in formal, quasiinstitutional settings, such examples of health education materials carry the message, by their location and form, that they contain useful information which is to be read by non-professionals. As documents which offer themselves up as "public," they offer advice-drawn presumably from authoritative sources-to all who will take it, and, in the true spirit of consumer education, are constructed and made available in ways which draw attention to their role as a mechanism for understanding complex and technical medical issues.

Moreover, in ways which I cannot explore here, such texts are also iconic of the discourse of the-physician-as-caring-technician, in that the documents seek not to judge, but to offer informed advice on medical issues, and as such are a tool for a patient interested in recovery, one who seeks to manage their dis-ease in accord with previously established medical norms.

Abjuring for the present a discussion of the semiotics of waiting room architecture in general and pamphlet placement in particular, we can ask: what is in the document? ${ }^{2}$ What does it say? The opening lines in the pamphlet (see Appendix 1) are instructive. Through these and subsequent lines I hope to show a series of social relations as they have become, if you will, textualized, and to provide support for the view that the discursive nature of medical practice is not incidental, but central to its social role. Chiropractic care, like much medical practice, is built upon a series of communicative acts which both constitute it and locate it within a larger social and political field. Medicine is in this view a way of speaking as much as it is a set of physical activities, or an institution; it has a rhetorical element to it which is essential, the thrust of which is to create and reproduce medicalized subjects.

Turning to the document, we can see that the initial move is to open up by "commending" the patient, who has reached out and sojourned into "unfamiliar territory." Addressed to the "new patient," the opening lines read, in part:

2 However, it could be noted that chiropractors' offices resemble other medical offices in that waiting for appointments in an anteroom is typical, and that sessions are not structured to encourage waiting after the session is over. This suggests that if such pamphlets are read, it would typically be prior to a session. As in most private medical settings, reading the material provided in the waiting room is the primary social activity. 
May we welcome you to this chiropractic office. You are to be commended for reaching out and venturing into a profession that may be unfamiliar to you. You may have been to some of the largest and most famous health institutions in our land. You may have spent large sums of money on various and often painful tests.

With these initial signifiers, the text adopts and invokes the image, presumably shared with the patient, of chiropractic as occupying a marginal place within the medical profession. The chiropractor is located within the realm of the medical in general, yet remains at the periphery of the specific institutional topography of the medical profession. The document anticipates that the practices at issue here (chiropractic care) are not well known, and signifies that they don't yet belong to an accepted medical community: they are foreign and so one is commended for engaging with them. The uncertain and contingent role of chiropractors is indicated pragmatically by the sentenceinitial use of the modal may in the opening line, its continued use in the rest of the first paragraph (where it occurs six times), and the frequent use of this and other modal indicators (will, may, if...then, must) in the remainder of the text. The use of may in the beginning indicates that the text secks to mark, not an epistemic uncertainty, but a deontic one (Cook and Suter, 1980). And the text immediately proceeds to locate that unfamiliarity, which is in fact the ill-defined role of the chiropractor, through a contrast with the well known: "the largest and most famous health institutions in our land."3

But these institutions are perhaps too well known (and may in fact be infamous). In the next sentences (the third and fourth in the initial paragraph) the text sets up a comparison, and evokes a judgement: at well known clinics patients spend large sums of money and submit to painful tests, activities which work directly against the presumption that such institutions heal. So while the initial move is to articulate a centre-periphery distinction, with chiropractic nearer to the margins, it is immediately made clear that sociocentral practices of medicine are themselves located at some distance from

3 The words suggest that the document was constructed using elements from American chiropractic literature: certainly the phrasc "most famous," "largest," and later on "expensive" (to the patient) are not adjectives usually associated with Canadian medical care. These words point to the presence of indicators of local knowledge in the text, but local knowledge which has been diffused broadly cnough to be salient to non-local (for example, non-American) rcaders. 
true practices of health care, and are themselves marginal with respect to appropriate methods of healing. ${ }^{4}$

And, in the fifth sentence of the text, a further element of the address to the patient unfolds, which supplements the first, the inability of conventional medicine to address disease appropriately, for such medicine is fixated with symptoms as opposed to cures: "...you may have learned the name that doctors have given to your problem, but the CAUSE of this problem may not yet have been found" (emphasis in original). So conventional medicine names but does not in the end penetrate the surface of the problem, resting content with a naive diagnosis of the appearance, its practices made blind by the limits of its medical ontology. It labours over the body but does not resolve disease. But of course this is familiar ground to the "new patient," who endures painful and fruitless tests; this presupposition is activated here in the text and briefly topicalized.

Over and against this familiar terrain stands the figure of the chiropractor, who is in unfamiliar territory, but who "welcomes you to the office." Like the characters who inhabit travelogues, the figures in the text (the patients) have not yet had constituted for them a proper place of healing; in their assigned role as travellers on a healing journey they leave behind the familiar (the famous health institutions), which no longer satisfies (because, in fact, patients don't get healed there), to go to places they have not been before in hope of uncovering there a hidden healing power. There they find, as with the innkeeper in Sterne's Tristram Shandy, that they are welcomed into that place by one who can rid them of their dis-ease, a disease which had only been exacerbated by the place from which they had begun their journey. In this textual manoeuvre, one of the classic tropes of travel writing, and for that matter ethnography (Clifford, 1988:159), is activated; the reader is drawn with the author into a secret knowledge, contained in a hidden place, with the entrance into the office doubling in the document as an initiation rite into a central and medically powerful truth.

So in these opening lines a context is set and a series of images invoked. Regarding context, I temporarily use a distinction drawn by Barthes (1972) between denotation and connotation, to note that at the denotative level the deictic pronoun "you" in the first sentence of the text is subsequently specified

4 I can only mark but not explore here the many different ways that "healing," "curing," "discasc," and "illness" arc understood by different social groups and different cultures. See Frankel, 1983; Locust, 1986; and Kleinman, 1980 for a discussion of some of these differences. 
as a certain kind of new patient; also denoted (as part of the context) is the "office" as the setting and the chiropractor as a "professional." In this regard, we might note the unresolved cohesion introduced by the (anaphoric) "we" in the first sentence, and the presupposition that the reader/addressee ("you") is in fact a "new patient." These create the effect of solidarity with the reader, an effect also found in oral folk narratives (Halliday and Hasan, 1976:298), which incidentally suggests that perhaps much shared knowledge of chiropractors' and physicians' roles is both folk and oral.

At the connotative level, the signs in the opening lines connote images of the traveller venturing into new territory to seek respite unavailable elsewhere; of the "office" as an established, formal, business-like location; and of the chiropractor as one whose calling involves specialized knowledge and long training: a "professional."

The contrast noted above between the world of conventional medicine and that of the chiropractic is further heightened by the next textual element of note: the enlarged title, which marks the break between the first and second orthographic paragraphs: "Chiropractic the Different Approach."

Here we encounter a peculiar thing: the sentence which follows this title, with its rhetorical question ("What can this doctor do for me?"), and its assumptions about the mental states of the reader ("the question uppermost in your mind"), seems to bear no syntactic (and only minimal lexical) relation to the title that hangs over it. This is what Agar has called a "breakdown in local coherence" (Agar, 1986; Agar and Hobbs; 1982). This occurs when contiguous or relatively contiguous elements of a text bear no straightforward relation of meaning to each other, and thus violate the maxim of relevance (Grice, 1975; Sperber and Wilson, 1986). What can the "question uppermost in your mind" have to do with "the different approach"? Prima facie, they seem to have no cohesive tie to each other, and thus do not form a coherent whole (Halliday and Hasan, 1976).

This local incoherence is further heightened by the next sentence in the second paragraph, which suggests, not difference, but similarity: "The reception room of the Doctor of Chiropractic seems to be quite similar to the offices of other doctors. There is a pleasant receptionist, an attractive room with professional type furniture..." Having signalled through the enlarged title hanging above it that the text is about difference ("chiropractic, the different approach"), we are presented instead with a similarity.

The local incoherence is temporarily resolved by the final clause in the sentence, which asserts that "there is a different approach to illness in the 
chiropractor's office"- different presumably from other doctors-thus reinforcing that it is difference that is at issue here, and further defining that difference. But while the theme of difference is raised again and its scope defined somewhat at the close of the paragraph (not a different setting, but a different approach), as yet the difference remains only loosely articulated at the surface of the text (we are left to ask, what is this difference?). The position of the chiropractor is only slowly beginning to form in the document, and has not yet emerged full blown from the workings of the text.

The paragraph which follows, the third in the document, deploys similar manoeuvres, employing themes introduced in prior parts of the text: it foregrounds first familiarity, then difference, then-a type of differencethe unexpected ("you will be asked questions...that you may not have considered important"). The document so far thus displays a strong thematic coherence, as the notion of difference/similarity is invoked and reiterated.

These textual manoeuvres-with the question of local coherence and the ill-defined invocations of difference and similarity-can be brought more into view when we consider the presuppositions required to make the encounter between the text and reader a socially meaningful affair. One such presupposition is that the readers of these texts share with the producers common schemata for interpreting conventional medicine. In these schemata, conventional medicine is focussed on consuming resources ("largest," "most famous," "large sums of money"); it treats symptoms (naming but not curing); and it reveals nothing not already known to the patient. It is content, in short, to label disease and extract from its presence fame and material resources, a kind of surplus value of disease which practitioners of conventional medicine accumulate.

These schemata acknowledge and further identify the practices of conventional medicine as practices of control, designed to be parasitic upon the body and to build upon its presence a structure of institutional authority. With this in mind the local incoherence noted above is dissolved, or at least subsumed, in a global coherence, which is the effort of the authors to favorably position chiropractic vis-à-vis conventional medicine.

We have not moved far into the document, but a significant amount of work has been done. I suggest that the document in these three paragraphs proceeds in a narrative form. The story is one of deprivation, dislocation, and a deficit both biological and epistemic. That is, the story is about a disease, compounded by medical doctors, a disease which can only get addressed by the introduction into the plot of a new and different element: a "chiropractic 
analysis," an analysis which does not rest upon the limited ontology of conventional (ineffective) medical practices. This new twist to the plot comes from the Doctor of Chiropractic, who welcomes you and "is interested in every detail of your history." In another classic trope of narrative fiction the introduction of new circumstances, or a new character (in this case the chiropractor) brings not only resolution to the initial deprivation, but also new knowledge to the protagonist.

The new location on the medical map created by chiropractic thus offers a view unimpeded by medical dogma: the body can better be surveyed and brought into order, its spine aligned just so, its deviations corrected, its parts brought back into harmony. I shall return to the fact that this place is not presented as entirely new in the document, but rather has ties to the discourse which undergirds conventional medical practices, and so does not attempt a clear break from or a complete repudiation of medical practice.

In these opening paragraphs, schemata are invoked and a position initiated which stands in contrast to the central elements of those schemata. Central to the text is the emergence of the figure of the "doctor of chiropractic." The chiropractor is both similar to and different from the other doctors (as yet unnamed but clearly recognized by the reader). In the next paragraph (the fourth in the document), there emerges from the workings of the text another character, "the new patient," to whom the pamphlet is addressed. Until now, the new patient has been identified (often implicitly) in the text as the victim/patient of other doctors, whose main attribute is a willingness to enter into the unknown. The text now makes explicit moves in the closing sentence of the fourth paragraph to construct the new patient, initially as an individual: "Each patient is an individual who must be studied and analyzed as such."

It is here that the global purpose of the pamphlet comes into its own. Up to now the central purpose of the pamphlet has been to orientate the reader to a "different" set of medical practices, a purpose which remains throughout the document. I suggest, however, that the text has an equally important role in attempting to constitute that "new patient," a role which emerges in this and subsequent paragraphs. For chiropractic needs more than just an ill body on which to lay its hands; it needs as well a subject category in which to place its potential patients; and it needs a discourse through which those bodies can become subject to its care, interpreted through its analysis, and made compliant with its dictates. And the text actively works to construct the reader as that patient; there is the frequent use of indexicals in the first 
three paragraphs, in particular the deictic pronouns "you" and "we," which, because they have no clear anaphoric reference act to presuppose (and, I suggest, construct), the reader as a potential chiropractic patient.

The sentence noted above ("Each patient is an individual who must be studied...as such") indicates that the "new patient" is to be constructed out of the old and familiar materials of possessive individualism. The insistence on the individuality of the patient, however, is soon negated by the rather categorical claim in the next paragraph, that no matter where your pain occurs, the chiropractor can show you how it starts in the spinal cord, more specifically, with "misaligned vertebrae":

Regardless of where your pain may be, you will usually find that the chiropractor can show you how it starts back in the spine, with misaligned vertebrae producing pressure on nerves....Yes, the answer may be found in your spine, where a surprising number of health problems originate.

The potential patient is indeed an individual, but is constituted in texts such as these as a very specific type of individual, one with a problem, presumably ill health of some sort, but also one who is understood as, the effect of a cause, hidden to many but known to the chiropractor: they are individuals whose most central structure is not in alignment. Given the degree to which, in chiropractic discourse, the spine and its parts are seen to penetrate and influence the whole, the misaligned vertebrae cannot in this state of disharmony co-exist peacefully with the remainder of the body, and must thus produce dis-ease. Thus the title heading this section: "Getting to the Cause of Your Problem."

Under this heading, the element of surprise again enters into the picture. In the second sentence of this paragraph, the categorical nature of the first claim ("Regardless...it starts back in the spine") is amplified by the explanation that a "surprising" number of health problems can be traced to the spine. Here again chiropractic asserts its right to be on terrain usually held by other health professionals. And it asserts this right by virtue of its ability to delve beneath the surface and get to the cause of the patient's difficulties, a cause which has heretofore escaped the attention of socio-central health institutions.

Halfway through this paragraph we encounter an interesting sentence: "This is basic neurology." In this rather blunt assertion we can detect a tension at the heart of the chiropractic discourse under analysis. There is, on the 
one hand, an avowed rejection of the institutions and practices of conventional medical care (expensive "famous health institutions," ineffective "doctors"). On the other hand the document signals (in this sentence and others) the reliance of chiropractic on the medico-scientific discourse that informs those conventional medical practices. "This is basic neurology" the document proclaims, as later it produces figures and diagrams which would rest comfortably in the anatomy section of a textbook on medical science. The document draws confidently upon scientific discourse to legitimate the status of chiropractic care. The suggestion seems to be, in fact, that chiropractic care is more authentic in some way than conventional medicine, as it is more in touch with the basics, it comes nearer to the roots of pathology, and it understands the body better, in a scientific way.

And it does so because it has a grasp of basic neurology, or, as implied by later diagrams, it knows better the central structures of the body, as these are revealed by medical science. But the contradiction, or at least the tension, between rejecting medical practices and accepting medical-scientific discourse is not foregrounded in the document. It lies hidden, and in its acceptance and valorization of medico-scientific discourse it shows its true social position, which is clearly within the parameters of medicine as a social practice, even while it rejects medicine as an institutional act. It is on the inside, looking in, hoping to dislodge the power of the centre. So if the effect of chiropractic discourse is to insert new subject categories (the chiropractor, and the chiropractic patient) into medical discourse, these subjects will be composed of elements drawn from the larger, dominant discourse of medical science, complete with the insistence that patients are to be studied and treated as individuals. Here is not the articulation of a new subjectivity, so much as the further specification and modification of previously established subject positions.

In the next few sentences there is a shift in the tone of the document, marked first by the penultimate sentence of the fifth paragraph: "This [the role of the spine] is best discovered by careful examination of the following illustrations," followed by the sentence, "Notice how the vertebrae are misaligned, with subsequent nerve pressure that misalignments are placing upon the nerve." The shift comes as the document moves in these sentences into instructional mode: the reader is now given, not merely information, but direction, in the form of (self-) diagnostic procedures to be followed, and is constituted as an active participant in the workings of the text. The shift is marked by less frequent use of indexicals, as the author(s) distances the text 
from a dependency on context, and by a reduced use of modals, which might through their indication of contingency compromise the authority of a central theme of chiropractic discourse, the signal role of the spine.

There are a number of possibilities raised by this shift. It may suggest the need on the part of chiropractors to overcome in forceful ways the dominant interpretation of illness, which would marginalize their work and valorize conventional medical practices. But the move into instructional mode may also suggest that the patient-subject specified through chiropractic discourse-and rendered into documentary form here-is a subject who suffers from not one, but two essential deficits. The patient is clearly one who is not at ease, who is ill and in need of care: this much has been established or mutually assumed early on in the text, and even prior to that, by virtue of the expected location of such documents. The patient is also, though, one who lacks a certain, crucial kind of knowledge. The patient being constituted through the text is in a similar position as those other doctors/health institutions, who can point to the disease but cannot establish its origin, who can verify problems but not causes. They do not know what the chiropractor can reveal, presumably through the "science of neurology," and the laying on of hands. Part of the job of the pamphlet is to remedy that knowledge deficit, and I suggest that it is an equally important job as the actual laying on of hands. So the move into the instructional mode is one way to satisfy the precondition for the work of the chiropractor, for one needs patients, chiropractic patients, in order to practice chiropractic.

It may be that both these factors come into play. In either case the tone of the text becomes increasingly imperative; the level of detail provided and the amount of documentary space used indicate that, for the authors of the text, instruction in the basics of chiropractic is of signal importance in overcoming other interpretations of pathogenesis.

Certain elements of the next few paragraphs of the document are also worthy of comment. Of note is the thematic coherence established by the sentences, in the sixth paragraph, "Every cell of your body receives nerve impulses directly or indirectly from the spine.... [i]s it any wonder that you are a 'bundle of nerves' when there is a misalignment in the spine?" This connects to the assertion, provided earlier in the text, that regardless of where a patient's pain is, "it usually starts back in the spine." These assertions have a double effect, both reiterating the centrality of the spine to the body, and the centrality of the chiropractor to curing illness. Again, in the seventh paragraph, the "inter-connectedness" and importance of the spine and its care- 
taker the chiropractor is heightened: the nerve that is attached to the spine is "a lifeline," and to irritate the nerve through a misaligned vertebra is to endanger the organ to which it is connected. "If no nerve impulse reaches the organ, it dies," whispers the text, in its attempt to establish through the spectre of death the critical role of the spinal column. The resolution for this unhappy possibility is provided in the next sentence, however, which speaks (tellingly, if one thinks of Foucault) of "normalizing" the nerve supply through a chiropractic adjustment, so as to avoid the pathologies associated with misaligned vertebrae.

The terms used here are interesting. One is "adjusted" by a chiropractor, and here again a difference from conventional medicine is articulated. While much of medical discourse adopts what Wulff has called a "demonic conception" of disease and disorder (Wulff, 1979), chiropractic discourse seems inclined more towards what might be called a "platonic perspective." In the demonic perspective, disease is an object present in the patient's body, and it consequently has its own identity as a pathological identity (Maseide, 1983; Wulff, 1979). Chiropractic discourse on the other hand, sees disorder and disease more as the result of an imbalance, a fatal misalignment, a failure of the parts to function in harmony. Rather than disease, one sees disorder in the body of the patient. And if the role of the (conventional) physician is to extirpate the pathological entity, the role of the chiropractor is to restore the unity of the whole, and to bring into balance the parts. The practice of chiropractic care (the adjustments, the "laying on of hands," the focus on the spinal column as both source and remedy for pathology) is inseparable from this metaphoric manner of understanding the body. This discourse of aligning the elements of the body through corrections to the spine is constitutive of chiropractic care.

These aspects of the document reveal a difference in the underlying medical metaphors which motivate the respective discourses of the physician and the chiropractor. But this difference at the level of underlying metaphors should not hide the similarity in the effects of the two discourses. Both work to make sensible the body upon which practitioners perform their operations, both work to create and sustain subject categories. And in a manner similar to physicians' discourse, chiropractic discourse requires that there be a docile body upon which to do its work, a body which is a product of a (modified) subject category: the chiropractic patient, with its individuality, its disorders, its distrust of conventional medicine, and its willingness to enter into unfamiliar territory. 
One final element of the document deserves our attention for the way in which it further specifies the patient. The concluding page of the document contains a text box with the enlarged title, "There are Three Types of Patients." The text beneath the heading provides a discussion of the three types, which, the document indicates, are derived from patients' willingness to continue with chiropractic care. The document suggests that the preferred type is one who continues "until their doctor dismisses them as completely recovered from their ailments," in contrast to one who "discontinue[s] chiropractic care prematurely." Without engaging in a lengthy comparative exercise with the written documents of conventional physicians, I suggest that the portentous tone of this piece would be out of place in the waiting rooms of more established medical professions, who are sufficiently central to the scheme of things that no such overt, rough typology is required. The need to classify patients and their pathologies is endemic to medical and scientific practice, but outside of a crude psychology the typology is not usually based on the patients' propensity to adhere to a particular course of treatment. The patients' acceptance of treatment in general is not an issue which appears with great frequency in the discourse of conventional physicians; that it does so in this pamphlet is an indication, again, of the uncertain status not only of chiropractic, but also of the chiropractic patient, which I suggest is still under formation as a subject category.

\section{Conclusion}

I have examined above a particular chiropractic document, in order to explore, in a preliminary and limited way, to be sure, the social location of chiropractors vis-à-vis their patients and other medical doctors, and to reveal the ways in which discourse subjects are textually constituted. The analysis suggests that chiropractic is not merely, as its etymology would suggest, a physical laying on of hands, but also-and just as critically—a way of speaking, a discourse, a series of communicative acts. And while those acts are perhaps produced initially by the chiropractor, they are surely also mutually accomplished in a series of encounters, both conversational and documentary, between the chiropractor, the patient, and unknown others. To miss this is to miss the way in which the subject categories which inhabit the medical realm are created, transmitted, modified, or rejected. It is also to misunderstand the nature of the chiropractor's work, which is as much to create a legitimate place for chiropractic and the accompanying docile bodies of potential patients as it is to remedy "spinal misalignments." 


\section{Appendix A}

The text of this Appendix $A$ is copyright (C) Parker Chiropractic Research Foundation, 1978. The three illustrations are approximations of those portrayed in the original document. Both text and illustrations are reproduced below solely as reference aids. The original document is produced in pamphlet form by the "Parker Chiropractic Research Foundation," and is distributed to chiropractors in Canada and the United States. A relatively limited examination of the documents in a number of chiropractors' offices indicates that the selected document is not untypical of the public health education information made available by chiropractors, although the actual disposition of such documents by chiropractors in medical encounters warrants further research. In the absence of detailed research on this, the analysis proceeds on the assumption that the document is roughly representative of key elements of chiropractic discourse, and that explanations of chiropractic have a role in the way chiropractors use discourse to accomplish social relations.

\section{To the New Patient}

May we welcome you to this chirpractic office. You are to be commended for reaching out and venturing into a profession that may be unfamiliar to you. You may have been to some of the largest and most famous health institutions in our land. You may have spent large sums of money on various and often painful tests. As a result, you may have learned the name that doctors have given to your problem, but the CAUSE of this problem may not yet have been found.

\section{Chirpropractic: The Different Approach}

"What can this doctor do for me?" is the question uppermost in your mind. The reception room of the Doctor of Chiropractic seems to be quite similar to the offices of other doctors. There is a pleasant receptionist, an attractive room with professional type furniture; but there is a different approach to illness in the chiropractor's office.

The Doctor of Chiropractic will use instruments for analysis that are familiar to you, and some different from those you have encountered before. You will be asked questions about falls and injuries that you may not have considered important. You will notice that the chiropractor is interested in every detail of your history, and will always take time to give complete atten- 
tion to your problem.

The Doctor knows that even though two patients may have the same disorder, they will not necessarily progress in the same manner. Each patient is an individual who must be studied and analyzed as such.

\section{Getting to the Cause of Your Problem}

Regardless of where your pain may be, you will usually find that the chiropractor can show you how it starts back in the spine, with misaligned vertebrae producing pressure on nerves that lead to where the pain is. Yes, the answer may be found in your spine, where a surprising number of health problems originate. You may have a misalignment of one or more spinal bones, called vertebrae. Those misalignments can then cause irritation or pressure on the nerves as they leave the spinal cord through openings between the vertebrae. This is basic neurology. This is best discovered through careful examination of the following illustration. Notice how the vetebrae [sic] are misaligned with subsequent nerve pressure that misalignments are placing upon the nerves.

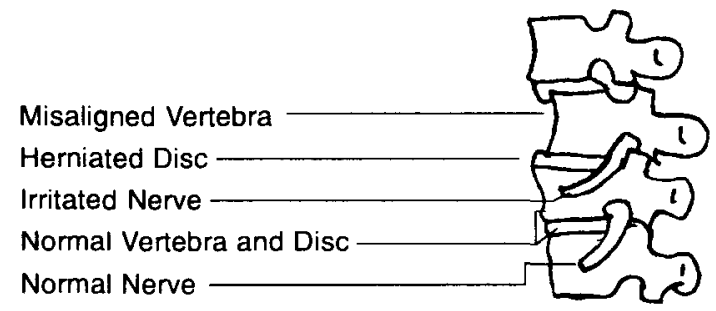

If you will look at the following charts, you will see the relationship of your spine to the various parts of your body. Every cell of your body receives nerve impulses either directly or indirectly from the spine. It is estimated that each one of these large nerve cables leaving your spine carries some 300,000 tiny nerves. Is it any wonder that you are a "bundle of nerves" when there is a misalignment in the spine? 
Chart of Autonomic Nervous System

Origins of nerves and areas affected

1 Thoracic section of spinal cord

2 Lumbar section of spinal cord

3 Superior cervical ganglion

4 Pupil of the eye

5 Blood vessels of the head

6 Salivary and lacrimal glands

7 Sympathetic trunk

8 Stellate ganglion

9 Heart

10 Lungs

11 Greater and lesser splanchnic nerves

12 Celiac ganglion

13 Stomach

14 Liver

15 Pancreas

16 Kidney

17 intestine

18 Superior mesenteric ganglion

19 Inferior mesenteric ganglion

20 Rectum

21 Bladder

22 Genitals

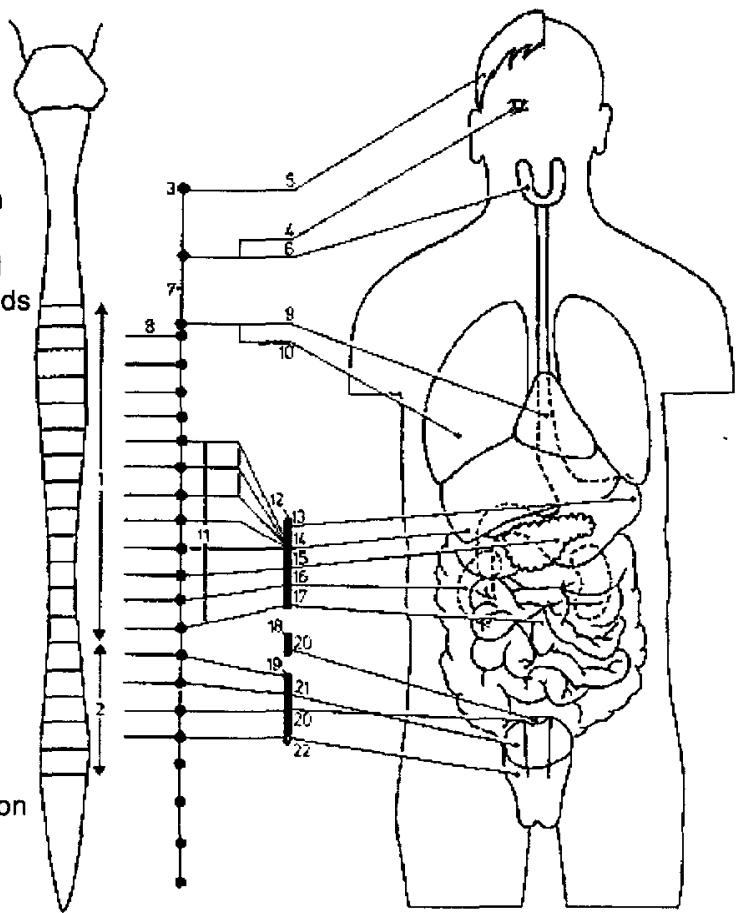

On the preceding chart locate the organ that is giving you discomfort. Trace the nerve from this organ back to the spine. This nerve is the lifeline of that organ. If no nerve impulses reach the organ, it dies. If a limited, or even increased, amount of impulses reaches it, the organ becomes sick. Normalize the nerve supply by a chiropractic adjustment, and normal function returns to the organ in exact ratio to the return of normal nerve impulses.

On the chart below, locate the area or areas of your body which are painful to you. Trace the nerves from these areas back to the spine. Pressure on these nerves by misalignment of the spine is likely to be the cause of your pain. 


\section{Chart of Spinal Nerve Distribution}

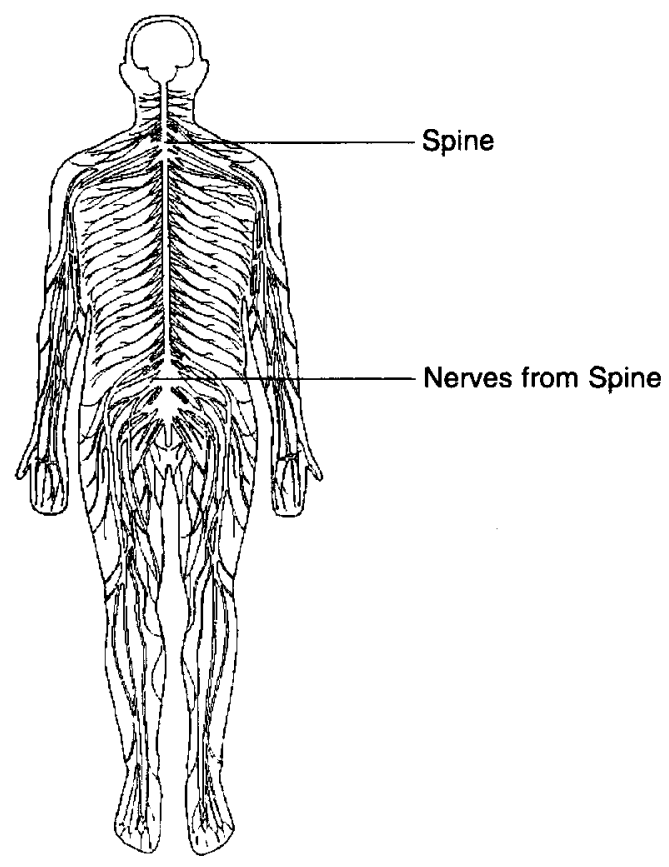

After taking $\mathrm{x}$-rays and completing various physical examinations and instrument checks, your chiropractor will be able to determine if spinal subluxation is the cause of your health problem. The examination results will give your doctor valuable information to aid in helping you get well. The x-rays will provide a blueprint for guidance in correcting the cause of your suffering.

Some healing methods strive to cover up, relieve, or deaden symptoms. Chiropractic finds and removes the cause. Once this has been done, the recuperative power of your body will build health.

\section{Will It Hurt?}

Perhaps you are wondering, "When the doctor finds a misalignment in my spine and removes it, will it hurt?" There is very little, if any, discomfort from a chiropractic adjustment, and it is relaxing to most.

The doctor uses very little force in making an adjustment. It is not unusual to see a one hundred pound doctor give a chiropractic adjustment to a two hundred pound patient with little effort. 


\section{Can Chiropractic Help?}

The Doctor does not attempt to determine this adjustment until at least a preliminary examination or, more often, a complete chiropractic examination has been made. After that you will be informed whether it is felt you can be helped by chiropractic. The Doctor has built a reputation on helping others without dangerous drugs or useless surgery. And a conscientious Doctor of Chiropractic will not accept your case unless it is felt there is a good chance of helping you. If there is only a fifty-fifty chance of helping you, you will be told, and the choice is left up to you.

\section{What about the Cost?}

No doubt you are also wondering what this is going to cost. This will vary depending on the severity of your condition and the type of care that is needed. Please feel free to make inquiries about fees before beginning treatment.

\section{How Long will It Take?}

How long will it take to recover from your ailment? The answer to this also varies with the circumstances of each individual case. The time it takes to recover will depend on the particular circumstances in your case. You can rest assured, however, that your Doctor of Chiropractic will get you well as quickly as possible.

Now that you have chosen chiropractic and have placed your health in the hands of a dependable Doctor of Chirorpractic, relax, cooperate, and give chiropractic a chance to help you to health.

\section{There are Three Types of Patients}

1. Some patients feel better after the first few adjustments, think they are well, and discontinue chiropractic care prematurely.

2. Some patients do not respond after a few more adjustments, get discouraged, and quit before they have a chance to begin recovery.

3. Fortunately, most patients, regardless of how long it takes, continue their adjustments until their doctor dismisses them as completely recovered from their ailments.

$35 \%$ of patients feel better within one week.

$35 \%$ within two weeks.

$20 \%$ within one month. 
$10 \%$ varies.

But remember!

Feeling Better Doesn't Mean All Problems Have Been Corrected. WHICH TYPE WILL YOU BE?

Merrijoy Kiner, Oswald Hall, Ian Coulter, Chiropractors, Do They Help? (sic) pp. 112-130, 134-138, 256. Fitzhenry \& Whiteside, Toronto, Montreal, Winnipeg, Vancouver, (1980).

F.P. De Giacomo, D.C., F.A.C.C., Man's Greatest Gift to Man...Chiropractic. pp. 52-60, 61-76, 180-181, LSR Learning Associates, Inc., Old Bethpage, NY, (1978).

Nathaniel Altman, The Chiropractic Alternative. pp. 27-32, 119, J.P. Tarcher, Inc., Los Angeles, CA, (1981).

D.D. Palmer, The Science, Ant and Philosophy of Chiropractic. pp. 528, 669, 781, Portland Printing House Co., Portland, OR, (1910).

\section{References}

Agar, M., and J. Hobbs. (1982). "Interpreting Discourse: Coherence and the Analysis of Ethnographic Interviews." In Discourse Processes. Vol. 5, pp. 132.

Agar, M. (1986). Speaking of Ethnography. Sage University Paper Series on Qualitative Research Methods, Vol. 2. Beverley Hills, Ca.: Sage.

Barthes, R. (1972). Mythologies. Trans. Annette Lavers. New York: Hill and Wang.

Battaglia, D. (1995). "Problematizing the Self: a Thematic Introduction." in Debbora Battaglia (Ed.) Rhetorics of Self-Making. Berkeley, Calif.:

University of California Press. pp. 1-15.

Bax, M. (1986). "Feelings for feelings: expressive information and its role in doctor-patient communication," in T. Ensick, A. van Essen, and T. van der Geest (Eds.), Discourse Analysis and Public Life: Papers of the Groningen Conference on Medical and Political Discourse. Dordrecht, Holland: Foris Publications. pp. 101-121.

Bourdieu, P. (1991). Language and Symbolic Power. Cambridge, Mass.: Harvard University Press. 
Candlin, C.M., and J. Lucas. (1986). "Interpretations and explanations in discourse: modes of 'advising' in family planning," in T. Ensick, A. van Essen, and T. van der Geest (Eds.), Discourse Analysis and Public Life: Papers of the Groningen Conference on Medical and Political Discourse. Dordrecht, Holland: Foris Publications. pp. 13-44.

Cicourel, A.V. (1987). "Cognitive and Organizational Aspects of Medical Diagnostic Reasoning." In Discourse Processes. Vol. 10, No. 4, pp. 347367. (1982). "Language and belief in a medical setting." In $\mathrm{H}$. Byrnes (Ed.), Contemporary perception of language: Interdistiplinary dimensions. Georgetown: Georgetown University Press.

Clifford, J. (1988). The Predicament of Culture. Cambridge, Mass.: Harvard University Press.

Collins, J. (1987). "Conversation and Knowledge in Bureaucratic Settings." In Discourse Processes. Vol. 10, No. 4, pp. 291-303.

Cook, S.J., and R.W. Suter. (1980). The scope of grammar. New York: McGrawHill Publishing Company.

Coulthard, M., and M. Ashby. (1976). "A linguistic description of doctorpatient interviews," in Michael Wadsworth and David Robinson (Eds.), Studies in everyday medical life. London: Martin Robinson and Co.

Erickson, F., and W. Rittenberg. (1987). "Topic control and Person Control: A thorny problem for foreign physicians in interaction with American patients." In Discourse Processes, Vol. 10, No. 4, pp. 401-415.

Fisher, S. (1983). "Doctor talk/patient talk: How treatment decisions are negotiated in doctor-patient communication." In S. Fisher and A.D. Todds (Eds.), The social organization of doctor-patient communications. Washington, D.C.: Centre for Applied Linguistics. pp. 221-241.

Frankel, R. (1983). "The laying on of hands: Aspects of the organization of gaze, touch, and talk in a medical encounter." In S. Fisher and A.D. Todds (Eds.), The social organization of doctor-patient communications. Washington, D.C.: Centre for Applied Linguistics. pp. 20-53. 
Freeman, S.H. (1987). "Organizational constraints as communicative variables in bureaucratic medical settings." In Discourse Processes. Vol. 10, No. 4, pp. 385-400.

Grice, H.P. (1985). "Logic and Conversation," in A.P. Martinich, (Ed.), The Philosophy Of Language. New York: Oxford University Press, pp. 159170.

Halliday, M.A.K, and R. Hasan. (1976). Cohesion in English. London: Longman.

Kleinman, A. (1980). Patients and healers in the context of culture: An exploration of the borderline between anthropology, medicine, psychiatry. Berkeley: University of California Press.

Locust C. (1985). American Indian Beliefs concerning health and unwellness. Washington: U.S. Department of Education.

Maseide, P. (1983). "Analytical aspects of clinical reasoning: A discussion of models of medical problem solving," in S. Fisher and A.D.Todd (Eds.) The Social organization of Doctor-Patient communication. Washington: The Centre for Applied Linguistics. pp. 241-265.

Silverman, David. (1987). Communication and medical practice: Social relations in the clinic. London: Sage Publications.

Silverman, K. (1983). The subject of semiotics. New York: Oxford University Press.

Sperber, D., and D. Wilson. (1986). Relevance: Communication and cognition. Cambridge, Mass.: Harvard University Press.

Waitzkin, H. (1976). "Information control and the micropolitics of health care." In Social Science and Medicine. 10, pp. 263-276. 